\title{
Author Correction: Leaf nutrients, not specific leaf area, are consistent indicators of elevated nutrient inputs
}

Jennifer Firn (1), James M. McGree, Eric Harvey @i), Habacuc Flores-Moreno (D), Martin Schütz, Yvonne M. Buckley (1D,

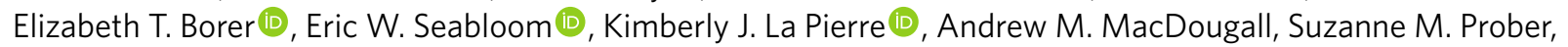

Carly J. Stevens, Lauren L. Sullivan, Erica Porter, Emma Ladouceur $\mathbb{D}$, Charlotte Allen, Karine H. Moromizato,

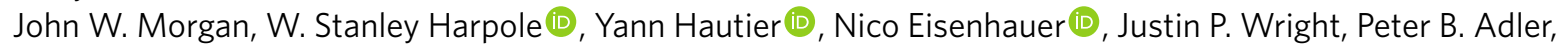
Carlos Alberto Arnillas, Jonathan D. Bakker, Lori Biederman, Arthur A. D. Broadbent, Cynthia S. Brown, Miguel N. Bugalho, Maria C. Caldeira, Elsa E. Cleland, Anne Ebeling, Philip A. Fay D, Nicole Hagenah, Andrew R. Kleinhesselink, Rachel Mitchell, Joslin L. Moore, Carla Nogueira, Pablo Luis Peri, Christiane Roscher, Melinda D. Smith (1), Peter D. Wragg and Anita C. Risch (1)

Correction to: Nature Ecology \& Evolution https://doi.org/10.1038/s41559-018-0790-1, published online 4 February 2019.

In the version of this Article originally published, there were unit conversion errors in the dataset when calculating specific leaf area (SLA) values at 10 of the 27 sites; errors were made when converting SLA from cm $\mathrm{g}^{-1}$ to $\mathrm{mm}^{2} \mathrm{~g}^{-1}$ and from $\mathrm{mm}^{2} \mathrm{mg}^{-1}$ to $\mathrm{mm}^{2} \mathrm{~g}^{-1}$. This resulted in two incorrect data points: Phleum pratense in plot 27 at the Frue.ch site (SLA $>300,000 \mathrm{~mm}^{2} \mathrm{~g}^{-1}$ ) and Poa secunda in plot 31 at the shps.us site (SLA $<60 \mathrm{~mm}^{2} \mathrm{~g}^{-1}$ ). These two values were changed to 'NA' in the dataset, and therefore some reported estimates were changed, resulting in changes to the data points in Fig. 2a, the top bar of Fig. 3 and several values in the text.

In the first paragraph of the Results and discussion section, the text 'We did, however, find evidence of a small but significant increase in SLA in the NP (mean $\log [\mathrm{SLA}]=8.79 \mathrm{~mm}^{2} \mathrm{~g}^{-1}$ ) and NPK fertilizer treatments (mean $\log [\mathrm{SLA}]=8.81 \mathrm{~mm}^{2} \mathrm{~g}^{-1}$ ) compared with the control (mean $\log [\mathrm{SLA}]=8.69 \mathrm{~mm}^{2} \mathrm{~g}^{-1}$ ), suggesting that' was corrected to 'We did, however, find evidence of a small but significant increase in SLA in the NP (mean $\left.\log [\mathrm{SLA}]=9.39 \mathrm{~mm}^{2} \mathrm{~g}^{-1}\right)$ and NPK fertilizer treatments $\left(\operatorname{mean} \log [\mathrm{SLA}]=9.41 \mathrm{~mm}^{2} \mathrm{~g}^{-1}\right) \mathrm{compared}$ with the control (mean $\left.\log [\mathrm{SLA}]=9.28 \mathrm{~mm}^{2} \mathrm{~g}^{-1}\right)$, suggesting that'. In the Fig. 2a caption, the text 'Intercept values $(8.7 \pm 0.6$ for a;' was changed to 'Intercept values $(9.3 \pm 0.3$ for $\mathbf{a} ;$ '.

In the first paragraph of the Results and discussion section, the text 'SLA showed the highest variability of any of the measured leaf traits at the site level (Fig. 3: 75\% of the variation in SLA in response to treatments was explained among sites) suggesting variation in SLA may be explained by other local abiotic and biotic factors not included in these models' was corrected to 'SLA showed a high variability at the site and species level (Fig. 3: 31\% of the variation in SLA in response to treatments was explained among sites and $\sim 47 \%$ among species), suggesting some variation in SLA may be explained by other local abiotic and biotic factors not included in these models'.

Original

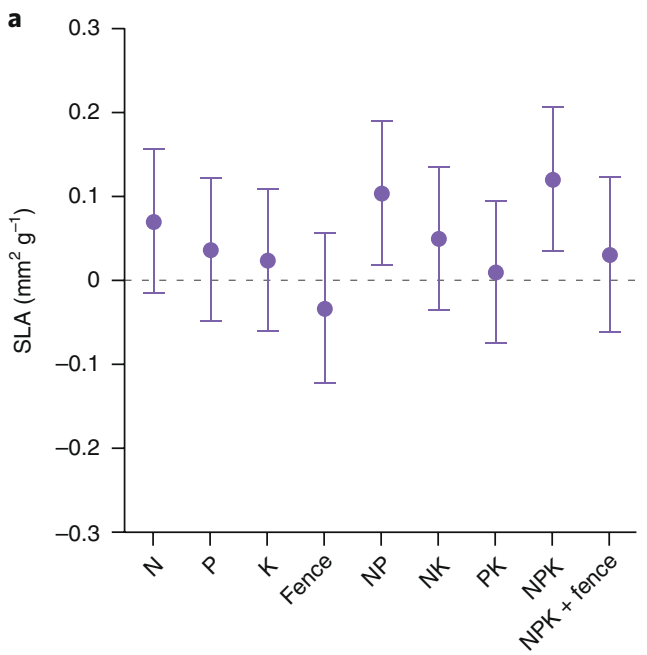

Corrected

a

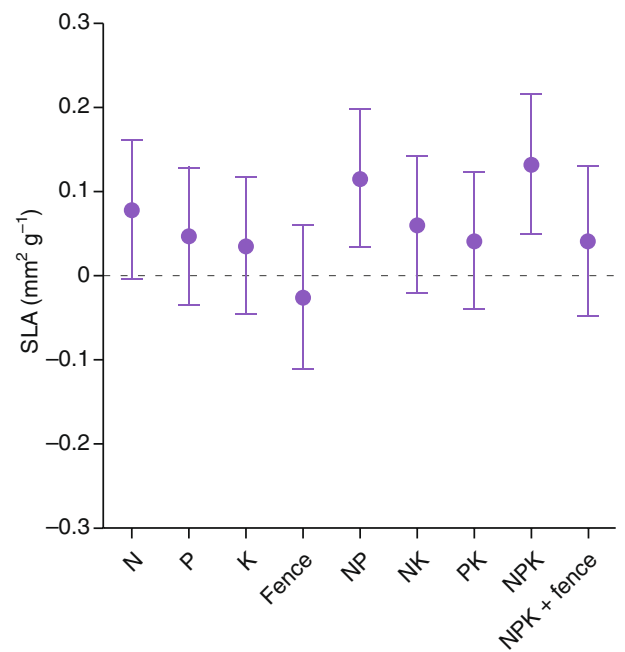

Fig. 2a | Original and corrected. 

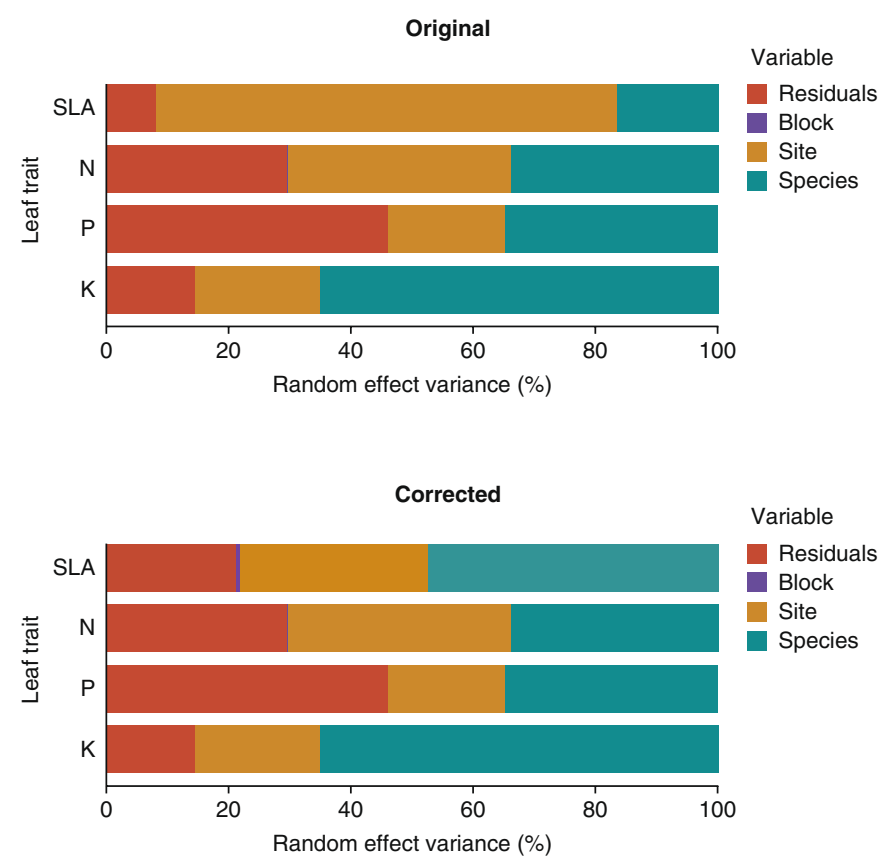

Fig. 3 | Original and corrected.

In the fourth paragraph of the Results and discussion section, the text 'leaf potassium had the highest $R^{2}$ value and SLA the lowest (leaf nitrogen, $R^{2}=0.53$; leaf phosphorus, $R^{2}=0.32$; leaf potassium, $R^{2}=0.55$; SLA, $R^{2}=0.11$ )' was corrected to 'leaf potassium had the highest $R^{2}$ value and SLA the lowest (leaf nitrogen, $R^{2}=0.54$; leaf phosphorus, $R^{2}=0.34$; leaf potassium, $R^{2}=0.57$; SLA, $R^{2}=0.22$ )'.

In addition, with the corrected SLA data, the standardized coefficient values for pathways have changed in the structural equation model (SEM) but overall qualitative findings have not changed. Four pathways in relation to SLA changed in the SEM: SLA was found to have a significant positive correlation with species temporal turnover (both in the meta-model (Supplementary Fig. 4) and in the final SEM outputs (Fig. 4 and Supplementary Fig. 5) and variability in mean annual precipitation, and a negative significant correlation with year of treatment. The prior significant negative correlation between SLA and soil potassium availability in the uncorrected data is no longer significant using the corrected values. These changes are reflected in the revised version of Fig. 4 and Supplementary Fig. 4 as well as the online interactive diagram, https://evidentlyso.com.au/clients/qut/functionalTraits $0170 /$. The following sentences in the text have been changed to reflect these corrections.

In the fifth paragraph of the Results and discussion section, the text 'Species temporal turnover was positively correlated with leaf nitrogen and potassium contents, but significant correlations were not found with the leaf phosphorus content or SLA' was corrected to 'Species temporal turnover was positively correlated with SLA and leaf nitrogen and potassium contents, but significant correlations were not found with the leaf phosphorus content'.

In the abstract, the sixth sentence 'Leaf nitrogen and potassium concentrations were positively correlated with species turnover, suggesting interspecific trait variation was a significant predictor of leaf nitrogen and potassium' was corrected to 'SLA, and leaf nitrogen and potassium concentrations were positively correlated with species turnover, suggesting interspecific trait variation was a significant predictor of these traits, but not of leaf phosphorus concentration. 


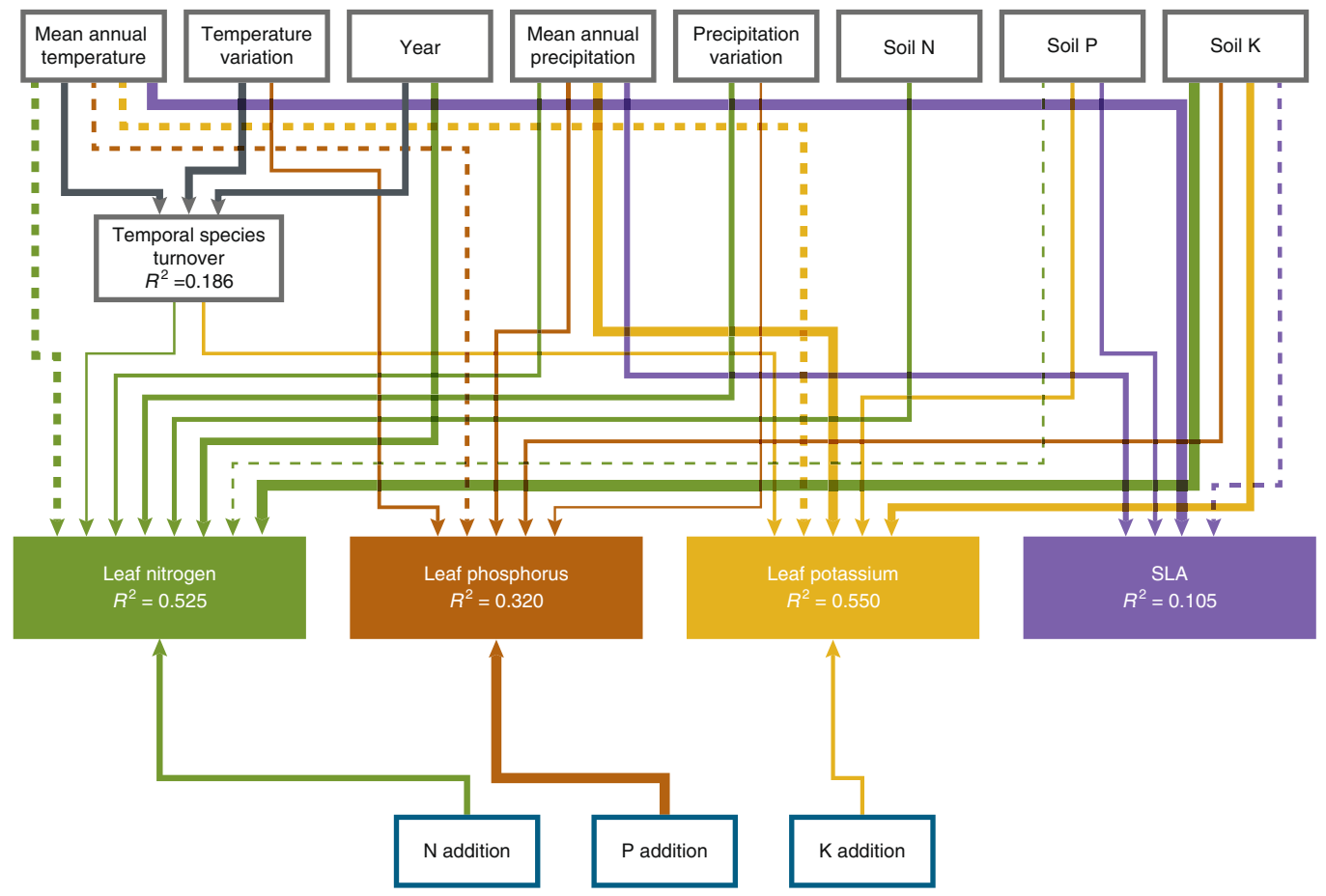

Strength of effect

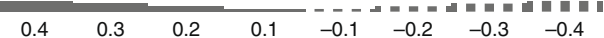

Fig. 4 | Original

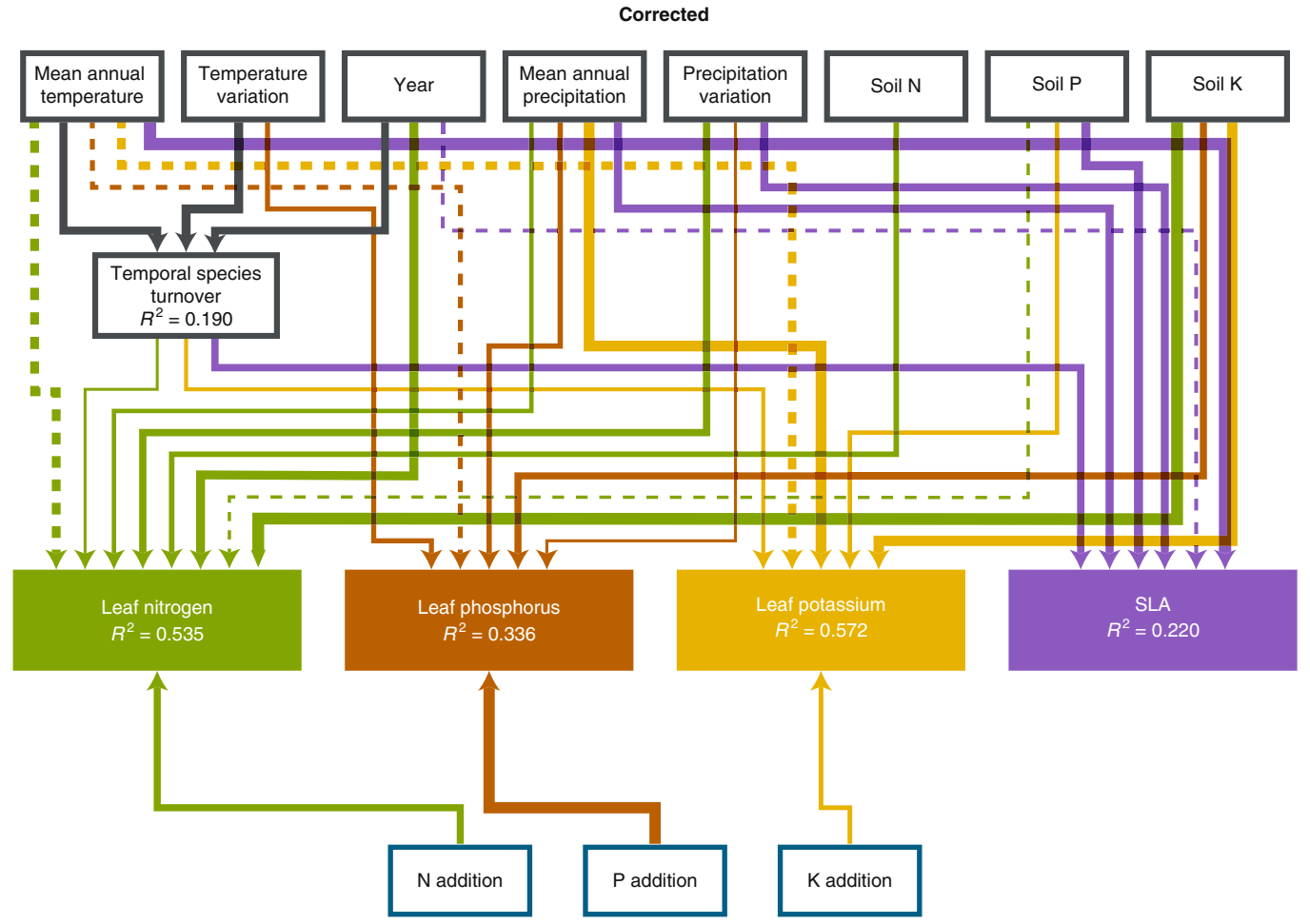

Strength of effect

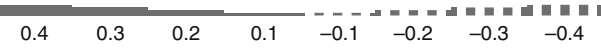

Fig. 4 | Corrected. 


\section{Original}

$$
\begin{aligned}
& \ldots--\rightarrow P>0.05 \\
& \longrightarrow P<0.05
\end{aligned}
$$

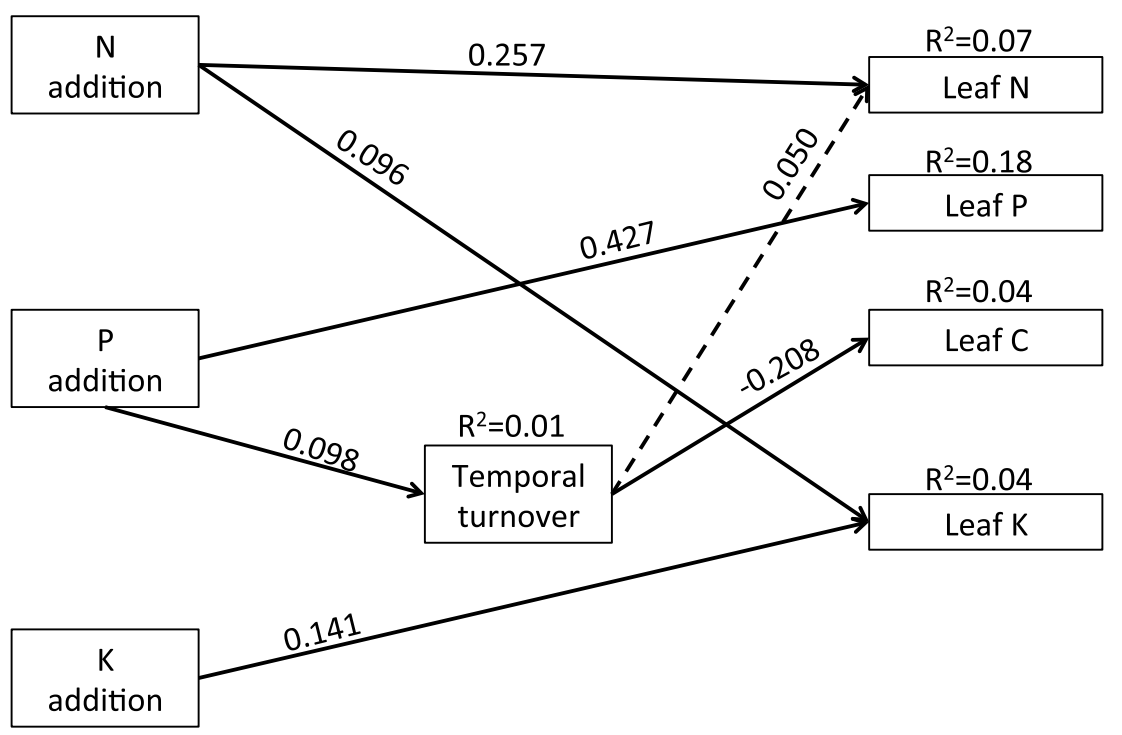

\section{SLA}

Corrected

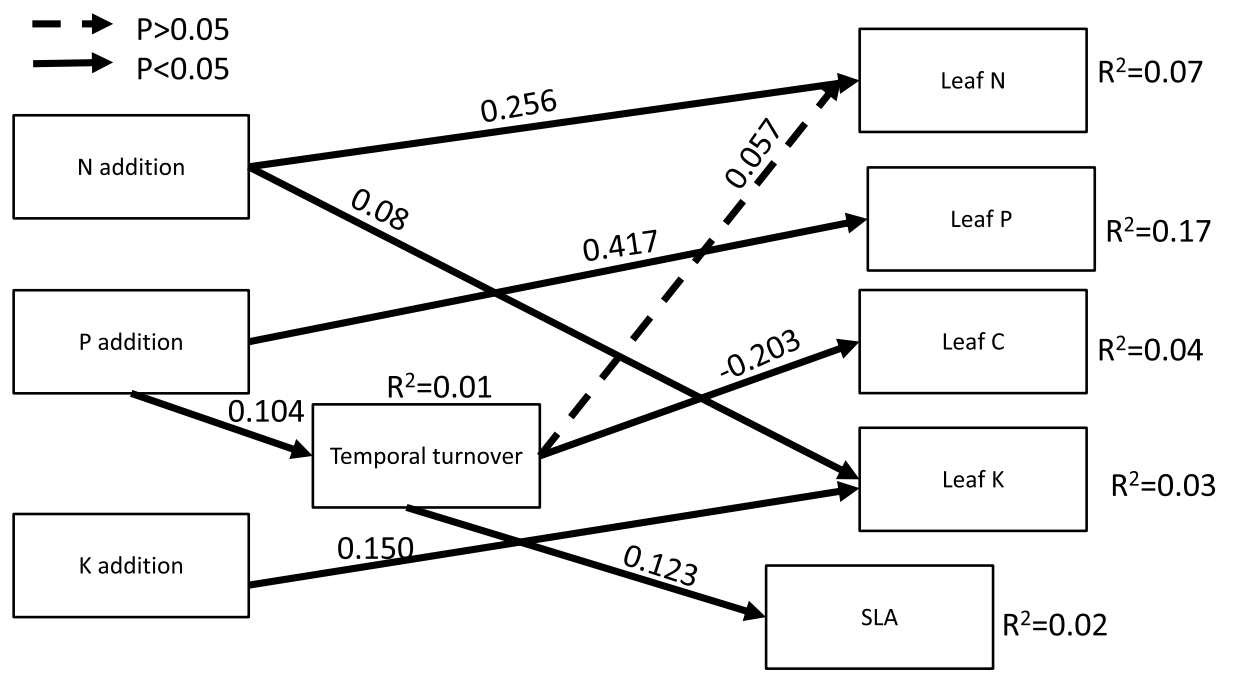

Supplementary Fig. 4 | Original and corrected. 


\section{Functional traits relationships}

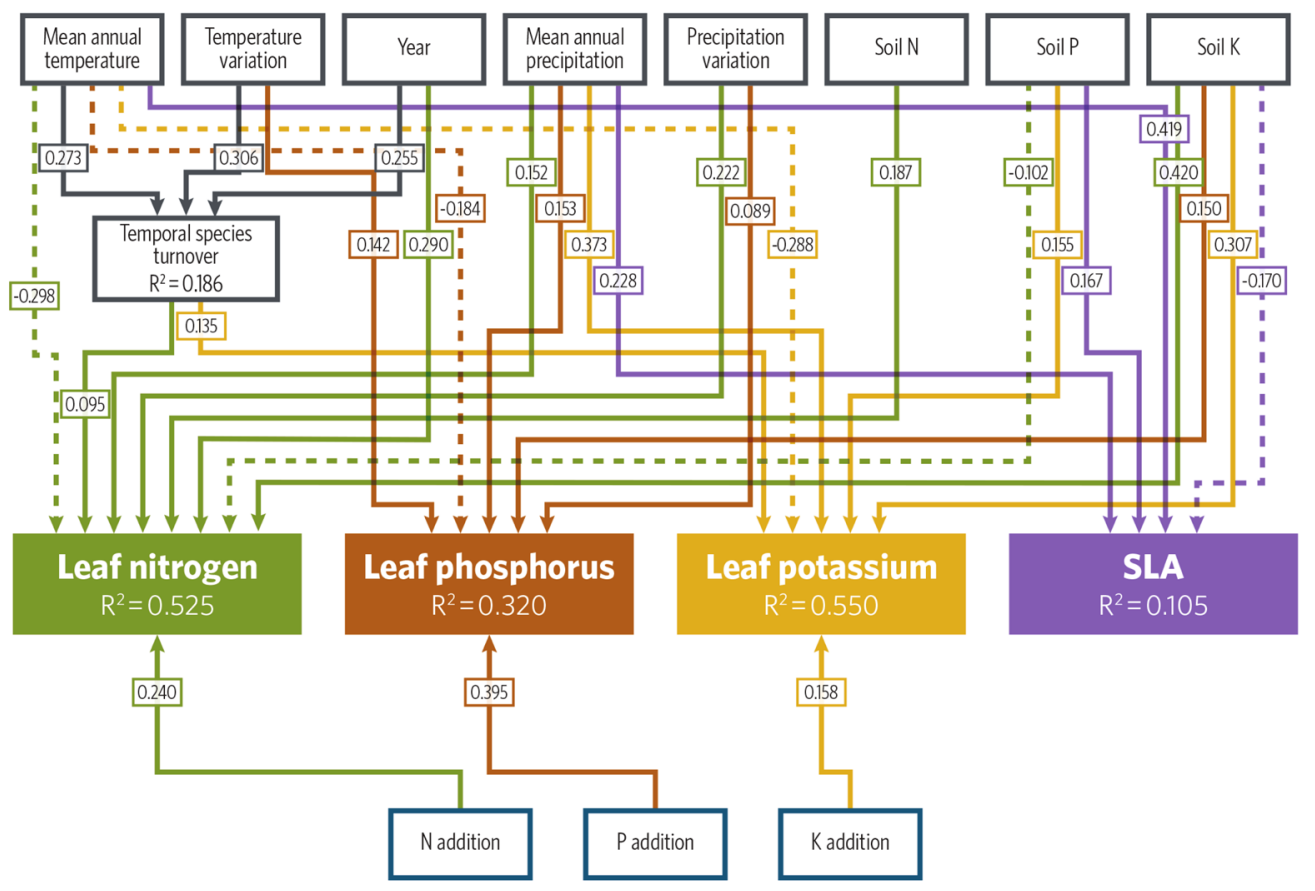

Supplementary Fig. 5 | Original.

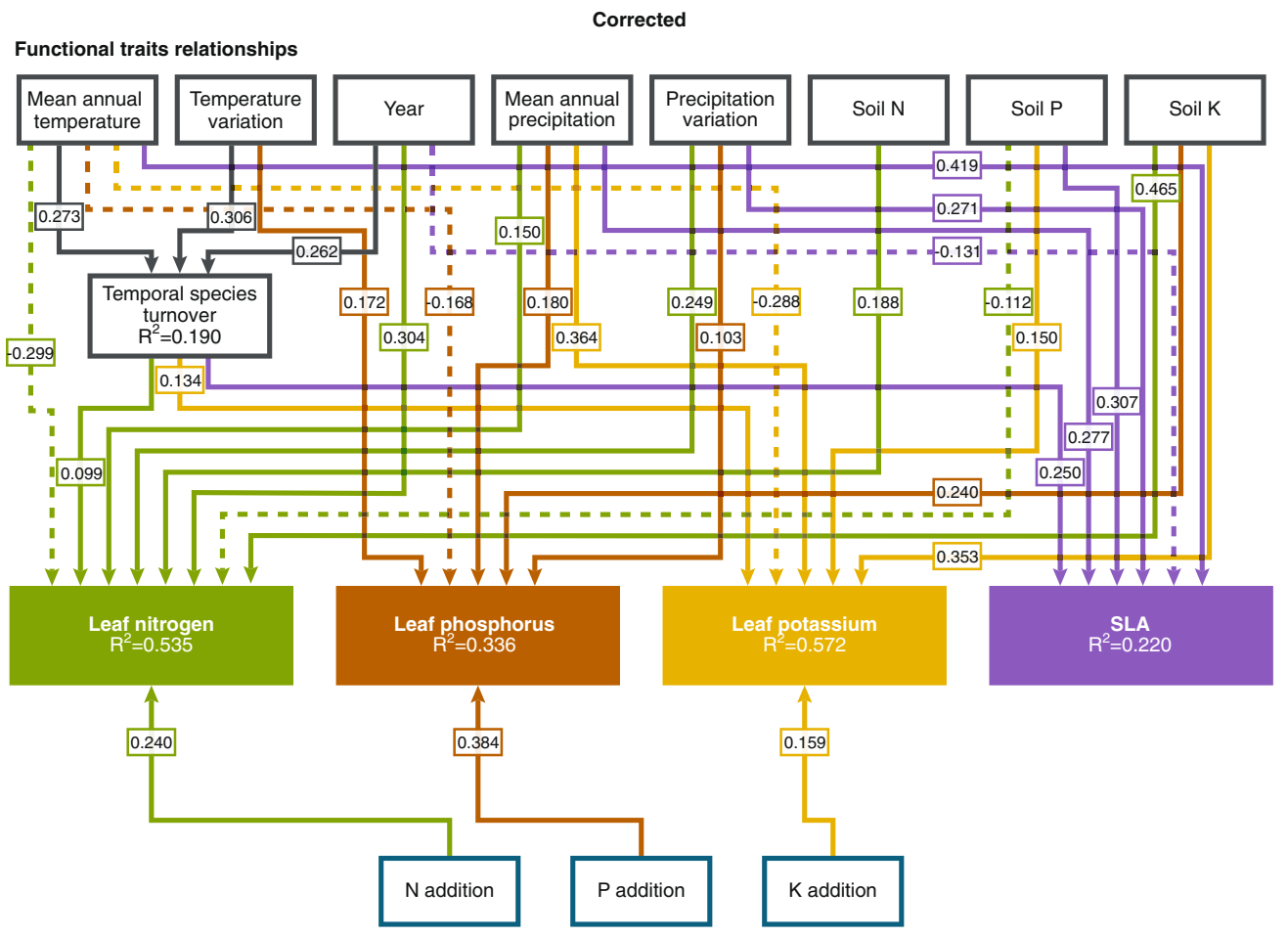

Supplementary Fig. 5 | Corrected. 
In Supplementary Table 2, co-variance values between leaf traits were also changed in the new model but the qualitative results remain the same. The original and corrected versions of Supplementary Table 2 are shown below.

Original

Supplementary Table 2: Co-variances between leaf traits based on the structural equation model.

\begin{tabular}{llll} 
& Leaf $\mathbf{P}$ & Leaf $\mathbf{K}$ & SLA \\
\hline Leaf $\mathrm{N}$ & 0.401 & 0.341 & 0.192 \\
Leaf $\mathrm{P}$ & & 0.462 & 0.224 \\
Leaf $\mathrm{K}$ & & & 0.153
\end{tabular}

\section{Corrected}

Supplementary Table 2: Co-variances between leaf traits based on the structural equation model.

\begin{tabular}{llll} 
& Leaf $\mathbf{P}$ & Leaf $\mathbf{K}$ & SLA \\
\hline Leaf $N$ & 0.438 & 0.344 & 0.176 \\
Leaf $\mathrm{P}$ & & 0.461 & 0.026 \\
Leaf $\mathrm{K}$ & & & 0.190
\end{tabular}

Furthermore, the data file on Dryad has been updated and can be found at the following link: https://doi.org/10.5061/dryad.qp25093.

We thank H.F.-M. (co-author), D. Blumenthal and P. Reich for drawing our attention to these issues in the SLA data.

Published online: 15 May 2020

https://doi.org/10.1038/s41559-020-1213-7

๑ The Author(s), under exclusive licence to Springer Nature Limited 2020 